\title{
PAPER
}

\section{Rotational lines of hydrogen in water}

To cite this article: Jacek Borysow et al 2019 J. Phys. B: At. Mol. Opt. Phys. 52095101

View the article online for updates and enhancements.

\section{IOP ebooks}

Bringing you innovative digital publishing with leading voices

to create your essential collection of books in STEM research.

Start exploring the collection - download the first chapter of every title for free. 


\title{
Rotational lines of hydrogen in water
}

\author{
Jacek Borysow ${ }^{1}\left(\mathbb{D},{\text { Massimo } \text { Moraldi }^{2} \text { and Martin Neumann }}^{3}\right.$ \\ ${ }^{1}$ Department of Physics, Michigan Technological University, Houghton, MI 49931, United States of \\ America \\ ${ }^{2}$ Dipartimento di Fisica e Astronomia, Università degli studi di Firenze, via G. Sansone 1, I-50019 Sesto \\ Fiorentino, Italy \\ ${ }^{3}$ Fakultät für Physik der Universität Wien, Strudlhofgasse 4, A-1090 Wien, Austria \\ E-mail: jborysow@mtu.edu
}

Received 16 November 2018, revised 6 March 2019

Accepted for publication 20 March 2019

Published 9 April 2019

\begin{abstract}
In this paper we report new calculations of the shape of the Raman rotational lines of hydrogen in solution with water. The method was based on a perturbative treatment and the calculations were performed for a system at room temperature and pressure $\approx 14 \mathrm{MPa}$, a thermodynamic state at which both measurements and accurate non-adiabatic calculations were available. The scope of the paper is to compare our numerical findings both with the computational results obtained with the nonadiabatic method and with the measured widths of the rotational lines. We found that our results are very similar to those obtained with the non-adiabatic method. Calculations of the widths were made with different models for the hydrogen-water intermolecular potential. The comparison of the numerical evaluations with the experimental findings allows us to judge how dependable the potential models are. The same calculations were performed also at larger pressures, up to $160 \mathrm{MPa}$. It was found that the widths of the rotational lines increased by increasing the pressure whereas, at the same pressure, they decreased when the rotational quantum number of the initial state was increased.
\end{abstract}

Keywords: Raman line-shape, gas liquid systems, intermolecular potentials

(Some figures may appear in colour only in the online journal)

\section{Introduction}

Raman spectroscopy of molecular hydrogen in water [1-6] is an important tool to understand both the interaction properties of water and hydrogen [4, 7-11] and the mechanisms by which a clathrate is formed [12].

Here, we will be concerned with anisotropic Raman spectroscopy $[1,4,13,14]$, and particularly with pure rotational lines $S_{0}(j)$, with $j$ indicating the angular momentum quantum number of the initial state of the Raman transition. The shape of these lines has been measured for both hydrogen and deuterium dissolved in water [3].

These line shapes contain information about all the interactions inside the solution, specifically about the hydrogen-water intermolecular potential. However, extracting information from the spectral profiles is not an easy task. Numerical evaluations of the rotational Raman line shapes have been performed in the past using a tentative intermolecular potential and various approximations: like static approximation, adiabatic approximation and the extreme motional narrowing limit of the Redfield theory [4]. Such approximations are, however, rather poor as was demonstrated by the comparison with results based on non-adiabatic numerical evaluations [13, 14]. The non-adiabatic calculations are more accurate but are also more cumbersome and time-consuming. Therefore, they are not appropriate when they are to be repeated to determine the effect on the line shapes of different hydrogen-water potential models. Thus it is desirable to search for an approximate method that is simple and, at the same time, able to reproduce the nonadiabatic results with reasonable accuracy.

In this paper we demonstrate that, in the case of solution of hydrogen in water, a rather simple method gives numerical results for the line widths that differ from the non-adiabatic one by no more than $10 \%$.

In our method we expand the logarithm of the polarizability correlation function in a perturbative series. In order to show the reliability of our approach, we compare the widths of the Raman rotational lines calculated with our method with those obtained by the non-adiabatic approach [13] and with the same intermolecular potentials. The comparison was made for a solution at room temperature and at a pressure of 
$\approx 14 \mathrm{MPa}$ for which both non-adiabatic calculations [13] and experimental measurements [1] are available.

The calculations were repeated here with two different $\mathrm{H}_{2}-\mathrm{H}_{2} \mathrm{O}$ potential models and the results were compared with the experimental findings [1].

Finally, we have calculated the widths of the rotational Raman lines also at higher pressures. We have found that the width of the lines increases with increasing pressure. Also, at each pressure, the width decreases by increasing the quantum number of the initial rotational state.

The paper concludes with a section about the discussion of the numerical results and comparison with the experiment.

\section{Theory}

In this section we will describe the theory that we have used to estimate the Raman rotational spectrum of molecular hydrogen in solution with water. To this scope we isolate a single hydrogen molecule ('radiating' molecule) and consider the time auto-correlation function of its anisotropic polarizability. The dynamics of the 'radiating' molecule is determined by its interaction with the rest of the solution, which is composed of all the water molecules plus the other hydrogen molecules.

In our theory, we will assume that the rotational Raman spectrum of molecular hydrogen in water can be written as a sum of isolated lines, each denoted by an initial and a final rotational state. Such an approximation is justified by the large separation between the resonance frequencies associated with the different rotational lines (see figure 3 in [1])

Accordingly we can write

$$
\begin{aligned}
S(\omega)= & \frac{4 \gamma^{2}}{9} \sum_{j_{i} j_{f}} P\left(j_{i}\right)\left(2 j_{i}+1\right) C\left(j_{i} 2 j_{f} ; 000\right)^{2} \\
& \times \frac{1}{2 \pi} \int d t \exp \left(-i\left(\omega-\omega_{i f}\right) t\right) C_{i f}(t),
\end{aligned}
$$

where $S(\omega)$ is Raman spectrum, $\gamma$ is the anisotropic part of the polarizability of the hydrogen molecule, $P(j)=$ $g_{j} \exp \left(-\beta E_{j}\right) / Z_{r o t}$ is the Boltzmann factor for the rotational state with quantum number $j$ and energy $E_{j}, g_{j}$ is the nuclear spin factor that equals 1 and 3 for even and odd $j$ 's respectively and, finally, $Z_{r o t}=\sum_{j} g_{j} \exp \left(-\beta E_{j}\right)(2 j+1)$ is the rotational partition function. Initial and final states of the Raman transitions are indicated by $i$ and $f$ and the transition frequency by $\omega_{\text {if }}[15]$.

Correlation functions $C_{i f}(t)$ can be expressed as $[16,17]$

$$
\begin{aligned}
& C_{i f}(t)=\frac{1}{\sum_{M}\left\langle\left\langle j_{i} M|U(-i \hbar \beta)| j_{i} M\right\rangle\right\rangle_{0}} \\
& \times \sum_{m m_{i} m_{i}^{\prime} m_{f}^{\prime} m_{f}^{\prime \prime}} C\left(j_{f} 2 j_{i} ; m_{f}^{\prime} m m_{i}^{\prime}\right) C\left(j_{f} 2 j_{i} ; m_{f}^{\prime^{\prime}} m m_{i}^{\prime^{\prime}}\right) \\
& \left\langle\left\langle j_{i} m_{i}|U(-i \hbar \beta)| j_{i} m_{i}^{\prime}\right\rangle\left\langle\left\langle j_{f} m_{f}^{\prime}\left|U^{\dagger}(t)\right| j_{f} m_{f}^{\prime \prime}\right\rangle\left\langle j_{i} m_{i}^{\prime^{\prime}}|U(t)| j_{i} m_{i}\right\rangle\right\rangle_{0},\right.
\end{aligned}
$$

where $U(z)=\exp \left(i z H_{0} / \hbar\right) \exp (-i z H / \hbar)[16,17]$ and $z$ is a complex variable. $H$ is the Hamiltonian of the system composed by all the water plus hydrogen molecules and $H_{0}$ is a Hamiltonian that equals the total Hamiltonian $H$ minus the part of the potential energy that depends on the orientation of the 'radiating' molecule. $H_{0}$ is considered here as the 'unperturbed' Hamiltonian. The internal states of the 'radiating' molecule are denoted as $|j m\rangle$, where $j$ and $m$ are the rotational quantum numbers of the 'radiating' molecule.

Finally, the symbol $\langle\ldots . .\rangle_{0}$ denotes a thermal average performed on the unperturbed system. The operator $V_{\text {pert }}=$ $H-H_{0}$ is treated as the perturbation.

The correlation function $C_{i f}(t)$ can be simplified in view of its perturbative expansion in powers of $V_{\text {pert }}$. Actually, it can be shown (see equation (2)) that, up to second order, the effect of $V_{\text {pert }}$ contained in $\left\langle j_{i} m_{i}|U(-i \hbar \beta)| j_{i} m_{i}^{\prime}\right\rangle$ amounts to an imaginary, antisymmetric correction to $\mathrm{C}_{i f}(t)$. Such a correction does not alter the total intensity of the line and is expected to be small. For such a reason we disregard its contribution and replace $\left\langle j_{i} m_{i}|U(-i \hbar \beta)| j_{i} m_{i}^{\prime}\right\rangle$ by $\delta_{m_{i} m_{i}^{\prime}}$.

Within such an approximation, the resulting correlation function takes the form

$$
\begin{aligned}
C_{i f}(t) \approx & \frac{1}{2 j_{i}+1} \\
& \times \sum_{m m_{i} m_{i}^{\prime} m_{f} m_{f}^{\prime} m_{f}^{\prime}} C\left(j_{f} 2 j_{i} ; m_{f} m m_{i}\right) C\left(j_{f} 2 j_{i} ; m_{f}^{\prime} m m_{i}^{\prime}\right) \\
& \left\langle\left\langle j_{f} m_{f}\left|U^{\dagger}(t)\right| j_{f} m_{f}^{\prime}\right\rangle\left\langle j_{i} m_{i}^{\prime}|U(t)| j_{i} m_{i}\right\rangle\right\rangle_{0} .
\end{aligned}
$$

Note that $C_{i f}(0)$ equals 1 according both to equation (2) and to its approximate form in equation (3).

Below, we define the potential $V$ for the interaction of the 'radiating' hydrogen molecule with the rest of the solution. Such a potential can be separated into components that differ for the symmetry with respect to the orientation of the 'radiating' molecule. Moreover, because we are interested in the pure rotational Raman spectrum of hydrogen, the intermolecular potential can be averaged over the ground vibrational state $(|0\rangle)$ of the 'radiating' molecule

$$
V(\hat{r}, \Gamma)=\sum_{L} \sqrt{\frac{4 \pi}{2 L+1}} \sum_{M} \bar{v}_{L M}(\Gamma) Y_{L M}(\hat{r}),
$$

where

$$
\bar{v}_{L M}(\Gamma)=\left\langle 0\left|v_{L M}(r, \Gamma)\right| 0\right\rangle .
$$

In equation (4), $L$ is a positive integer and the sum runs from $L=0$ to infinity. $r$ and $\hat{r}$ are the modulus and the direction of the vector joining the two nuclei in the 'radiating' hydrogen. Finally, $\Gamma$ represents all the coordinates defining the configuration of the solution minus the roto-vibrational coordinates of the 'radiating' molecule.

As already stated before, the perturbation is the part of the $\mathrm{H}_{2}-\mathrm{H}_{2} \mathrm{O}$ potential that depends on the hydrogen orientation, that is

$$
V_{\text {pert }}(\hat{r}, \Gamma)=\sum_{L>0} \sqrt{\frac{4 \pi}{2 L+1}} \sum_{M} \bar{v}_{L M}(\Gamma) Y_{L M}(\hat{r}) .
$$


Next, we applied perturbation theory to the operators $U(t)$ and $U^{\dagger}(t)$ and finally obtain $C_{i f}(t)$ correct up to second order in $V_{\text {pert }}$ according to the equation

$$
C_{i f}(t)=\exp \left(-\frac{1}{\hbar^{2}} \int_{0}^{t} d t^{\prime} \int_{0}^{t^{\prime}} d t^{\prime \prime} K_{i f}\left(t^{\prime \prime}\right)\right)
$$

with

$$
\begin{aligned}
K_{i f}(t)= & \sum_{L \neq 0}\left[\sum _ { j } \left(C\left(j_{f}, L, j: 000\right)^{2} \exp \left(-i \omega_{j_{f} j} t\right)\right.\right. \\
& \left.+C\left(j_{i}, L, j: 000\right)^{2} \exp \left(i \omega_{j_{i} j} t\right)\right) \\
& -2 C\left(j_{i} L j_{i} ; 000\right) C\left(j_{f} L j_{f} ; 000\right) \\
& \left.\times\left(\left(2 j_{i}+1\right)\left(2 j_{f}+1\right)\right)^{1 / 2}\left\{\begin{array}{ccc}
L & j_{i} & j_{i} \\
2 & j_{f} & j_{f}
\end{array}\right\}\right] \chi_{L}(t)
\end{aligned}
$$

and $\chi_{L}(t)$ the time correlation function of the anisotropic components of the potential energy of $\mathrm{H}_{2}$ in solution with water

$$
\chi_{L}(t)=\frac{1}{2 L+1} \sum_{M}\left\langle\left\langle\bar{v}_{L M}(\Gamma(t)) \bar{v}_{L M}(\Gamma(0))^{*}\right\rangle_{0} .\right.
$$

It is to be noticed that $K_{i f}(t)$ is a complex function, with the real part symmetric and the imaginary part antisymmetric with respect to time $t$. The antisymmetric part does not contribute to the total intensity of the line. In principle it can introduce non symmetric terms in the spectrum but these are expected to be small and as of to date have not been observed. For these reasons we have neglected these contributions to the spectrum and have retained only the real part of the correlation function $K_{i f}(t)$

$$
\begin{aligned}
K_{i f}(t)= & \sum_{L \neq 0}\left[\sum _ { j } \left(C\left(j_{f}, L, j: 000\right)^{2} \cos \left(\omega_{j_{f} j} t\right)\right.\right. \\
& \left.+C\left(j_{i}, L, j: 000\right)^{2} \cos \left(\omega_{j_{j} j} t\right)\right) \\
& -2 C\left(j_{i} L j_{i} ; 000\right) C\left(j_{f} L j_{f} ; 000\right) \\
& \left.\times\left(\left(2 j_{i}+1\right)\left(2 j_{f}+1\right)\right)^{1 / 2}\left\{\begin{array}{ccc}
L & j_{i} & j_{i} \\
2 & j_{f} & j_{f}
\end{array}\right\}\right] \chi_{L}(t) .
\end{aligned}
$$

Among all the anisotropic components in equation (4), $L=2$ is particularly important because at large distances it can be represented as the interaction of the $\mathrm{H}_{2}$ electric quadrupole with the water dipole moment

$$
\bar{v}_{2 M}=\langle 0|Q(r)| 0\rangle \sum_{a} \frac{q_{a}}{R_{a, c m}^{3}} \sqrt{\frac{4 \pi}{5}} Y_{2 M}^{*}\left(\hat{R}_{a, c m}\right),
$$

where the index $a$ refers to the nuclei of all the water molecules and $q_{a}$ is the charge associated with the nucleus $a$ according to the simple point charge model for the waterwater interaction of Berendsen et al [18]. $\langle 0|Q(r)| 0\rangle$ is the quadrupole moment of hydrogen averaged over the ground vibrational state.

In previous calculations $[4,13]$ the value $0.466 e a_{0}^{2}[19]$ was used for $\langle 0|Q| 0\rangle$. Here we have used both that value and the more recent value $\langle 0|Q| 0\rangle=0.485 e a_{0}^{2}$ which is taken from [20] (the value used here is actually $1 / 2$ of the value appearing in [20] because of a different definition of the quadrupole moment).

\section{Molecular dynamics results}

In order to determine the shape of the Raman lines, it is necessary to compute the correlation function $\chi_{2}(t)$ of equation (9). The components $\bar{v}_{2 M}(\Gamma)$ are defined in equation (11) and the explicit expression for correlation function is

$$
\begin{aligned}
& \chi_{2}(t) \\
& =\frac{1}{5}\langle 0|Q| 0\rangle^{2} \sum_{a a^{\prime}}\left\langle\frac{q_{a}}{R_{a, c m}^{3}(0)} \frac{q_{a^{\prime}}}{R_{a^{\prime}, c m}^{3}(t)} P_{2}\left(\hat{R}_{a, c m}(0) \cdot \hat{R}_{a^{\prime}, c m}(t)\right\rangle_{0},\right.
\end{aligned}
$$

where $a$ and $a^{\prime}$ represent the positions of all the nuclei in the water molecules. $P_{2}$ is Legendre polynomial and $R_{a, \mathrm{~cm}}$ and $\hat{R}_{a, \mathrm{~cm}}$ are modulus and unit vector of $\vec{R}_{a, \mathrm{~cm}}$, respectively. The vector $\vec{R}_{a, c m}$ originates at the center of mass of the hydrogen molecule and ends at the nucleus $a$ of the water molecule. Finally, $q_{a}$ is the charge located at the site of the nucleus $a$ of the water molecule [18].

We have performed the calculations with two different values for $\langle 0|Q| 0\rangle, 0.466 e a_{0}^{2}$ [19] and $0.485 e a_{0}^{2}$ [20]. We have computed the correlation function $\chi_{2}(t)$ by means of classical molecular dynamics. In these calculations the water molecules are treated as rigid (their vibrations are not taken into account). They interact among themselves by means of the Simple Point Charge potential of Berendsen et al [18]. For the hydrogen-hydrogen potential we take a Lennard-Jones model based on the Silvera and Goldman potential [21]. Finally, the $\mathrm{H}_{2}-\mathrm{H}_{2} \mathrm{O}$ interactions are determined by the potential energy $\bar{v}_{00}(\Gamma)$ from equation (5). Accordingly, the system is composed of water molecules that can translate and rotate plus hydrogen molecules that can only translate.

The computations of $\chi_{2}(t)$ have been performed with two different Lennard-Jones models for the isotropic $\bar{v}_{00}$ component of the hydrogen-water potential. The Lennard-Jones models depend on the distance between the center of mass of the $\mathrm{H}_{2}$ molecule and the position of the oxygen in the water molecule. The parameters $\left(\sigma=2.92 \AA, \epsilon=1.139 \mathrm{~kJ} \mathrm{~mol}^{-1}\right)$ of first one, named hereafter $V_{\text {Hunter }}$, are taken from gas phase scattering data [22]. $V_{\text {Hunter }}$ has been used in the past [4, 22] to calculate the widths of the rotational lines, both in the adiabatic [4] and non-adiabatic [13] approximations. The parameters $\left(\sigma=3.10 \AA, \epsilon=0.431 \mathrm{~kJ} \mathrm{~mol}^{-1}\right)$ of the second potential, named $V_{\text {Alavi }}$, are derived from the application of the Lorentz-Bertholet combination rules [7].

Molecular dynamics simulations have mostly been performed in the isokinetic variant of the canonical NVT ensemble. The density of water in the solution is determined with the help of the results obtained in [6] and according to the procedure described in [23]. The calculations that have been performed with 432 water molecules and 1 hydrogen 


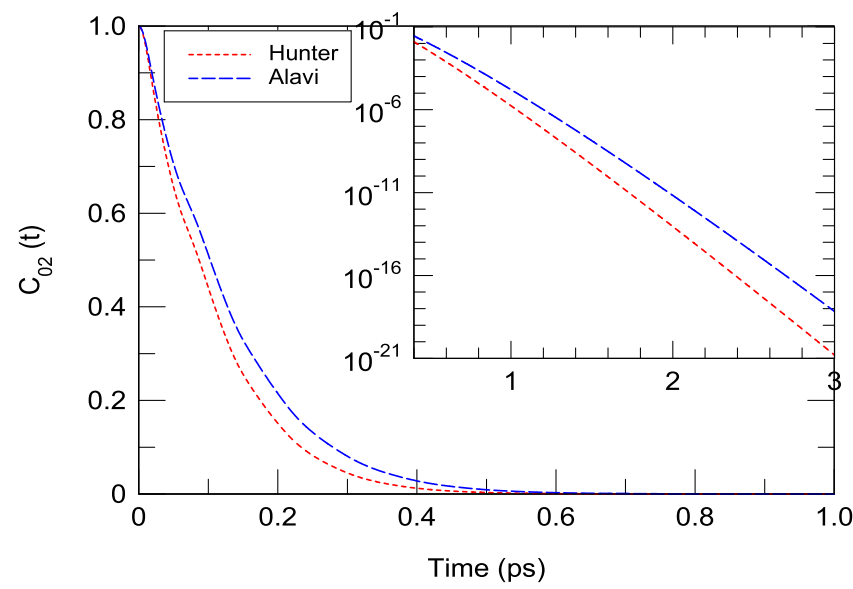

Figure 1. $C_{02}(t)$ with potential models $V_{\text {Hunter }}$ and $V_{\text {Alavi }}$ and $Q=0.485 e a_{0}^{2}$.

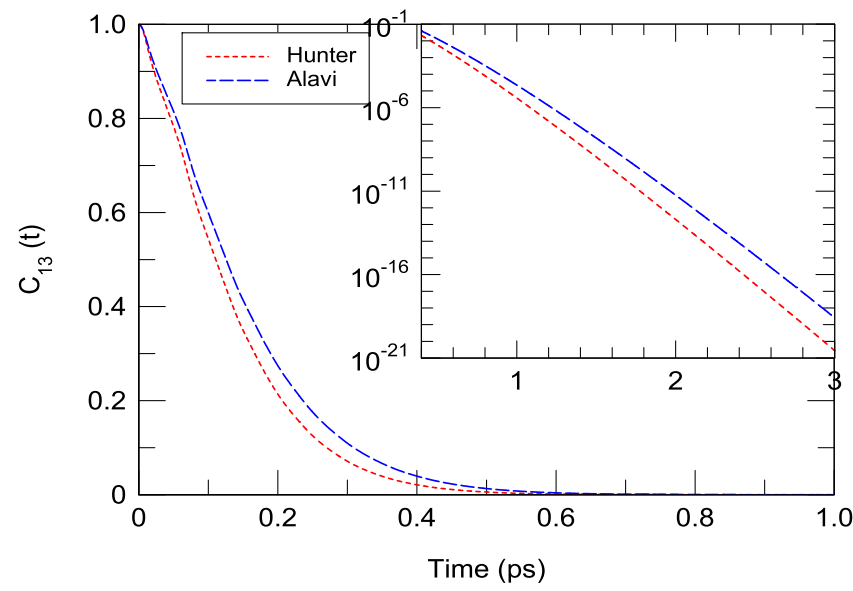

Figure 2. $C_{13}(t)$ with potential models $V_{\text {Hunter }}$ and $V_{\text {Alavi }}$ and $Q=0.485 e a_{0}^{2}$.

molecule were compared with the experiment. According to solubility measurements $[5,6]$, such a ratio corresponds to a pressure of $14.7 \mathrm{MPa}$ at $T=280 \mathrm{~K}$ [23] which is close to the experimental conditions [1]. Additionally, we computed the correlation functions also for 5 and 9 hydrogen molecules surrounded by 432 water molecules.

We have chosen the NVT ensemble rather than the NPT one for the sake of simplicity. However, in order to estimate the differences due to the use of either the NVT or the NPT ensemble, we have performed NPT simulations of pure water at the pressures of 15,80 and $160 \mathrm{MPa}$ and determined the corresponding densities. Such densities agree with the experimental ones within an uncertainty of $0.5 \%-1 \%$.

Both isokinetic and Nose-Hoover canonical ensembles have been employed in order to check any differences in resulting correlation functions. For the basic simulation cell, a rhombic dodecahedron one has been used. Also, a rather accurate Ewald sum has been used to properly take into account the long range electrostatic interactions. The time steps were $0.001 \mathrm{ps}$ and the runs $10^{7}$ time steps (10 ns) long. In the Nose-Hoover simulations, the relaxation time was 1 ps.

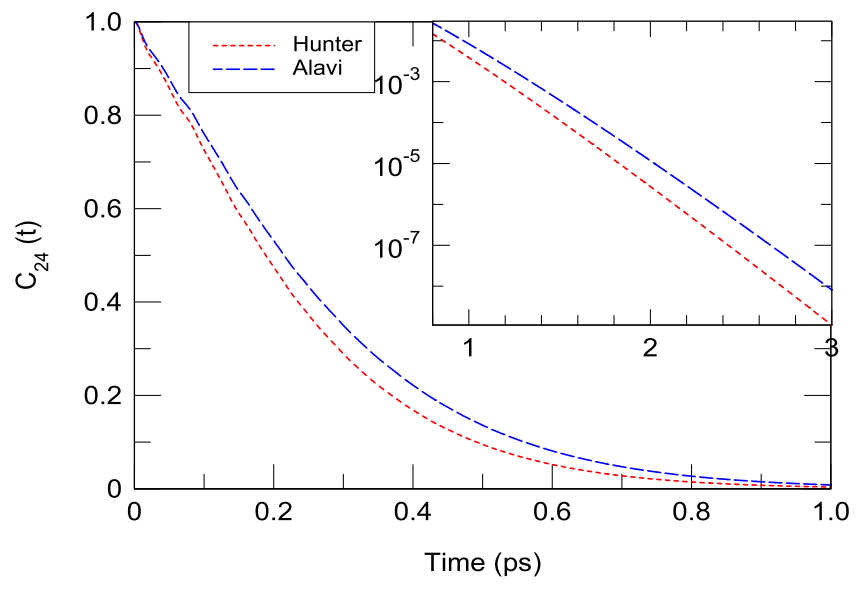

Figure 3. $C_{24}(t)$ with potential models $V_{\text {hunter }}$ and $V_{\text {Alavi }}$ and $Q=0.485 e a_{0}^{2}$.

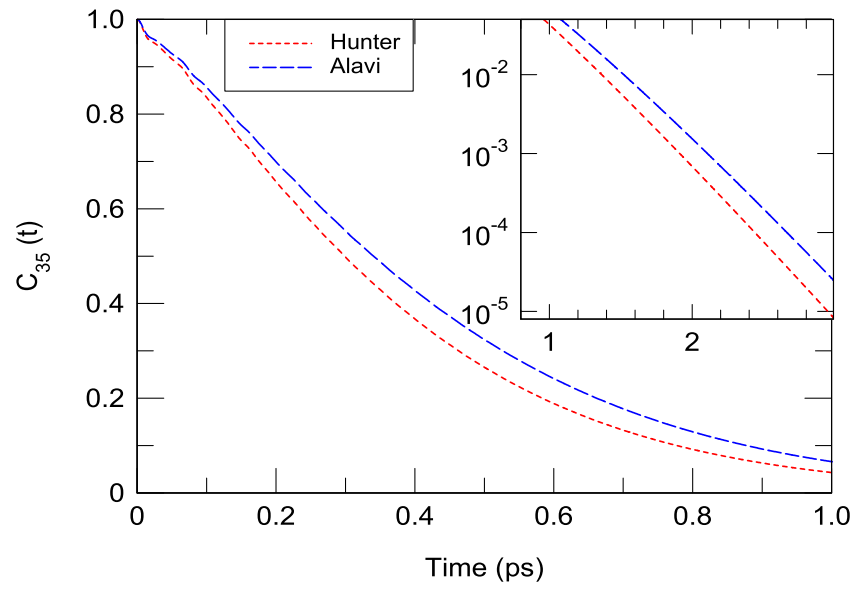

Figure 4. $C_{35}(t)$ with potential models $V_{\text {hunter }}$ and $V_{\text {Alavi }}$ and $Q=0.485 e a_{0}^{2}$.

We have computed the correlation functions $C_{i f}(t)$ for two different $\bar{v}_{00}$ models. Results are shown in figures 1-4. Correlation functions were calculated using both model potentials $V_{\text {Hunter }}$ [4] and $V_{\text {Alavi }}$ [7] for $\bar{v}_{00}$. The decay time of the correlation function increases when increasing the initial rotational quantum number and thus the rotational frequency. In figures 5-8 we report the Fourier transforms of the correlation functions $C_{i f}(t)$ that are denoted by $I_{i f}(\omega)$. They show the line shapes of the different rotational Raman lines and their dependence on the choice of the model for $\bar{v}_{00}$. The integrated intensity of all the line shapes in figures 5-8 are normalized to unity.

The numerical results reported in figures 1-8 have been calculated at the relative density $1 / 432$ with hydrogen quadrupole moment equal to $0.485 e a_{0}^{2}$ [20].

\section{Comparison with experiments and previous calculations}

In this section we report the numerical results for the Half Width at Half Maximum (HWHM) for the lines $S_{0}(j)$ with 


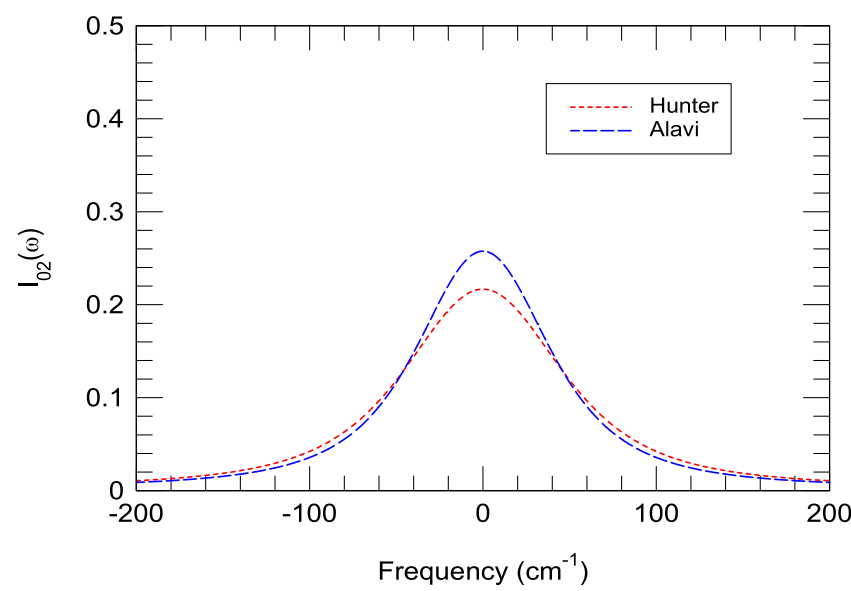

Figure 5. Fourier transform of $C_{02}(t)$, denoted here as $I_{02}(\omega)$, computed with potential models $V_{\text {hunter }}$ and $V_{\text {Alavi }}$ and $Q=0.485 e a_{0}^{2}$.

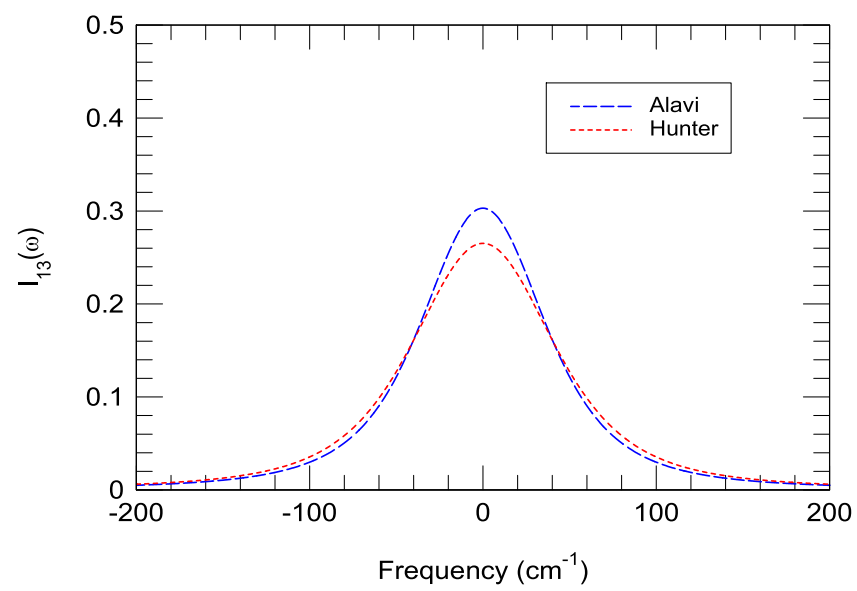

Figure 6. Fourier transform of $C_{13}(t)$, denoted here as $I_{13}(\omega)$, computed with potential models $V_{\text {hunter }}$ and $V_{\text {Alavi }}$ and $Q=0.485 e a_{0}^{2}$.

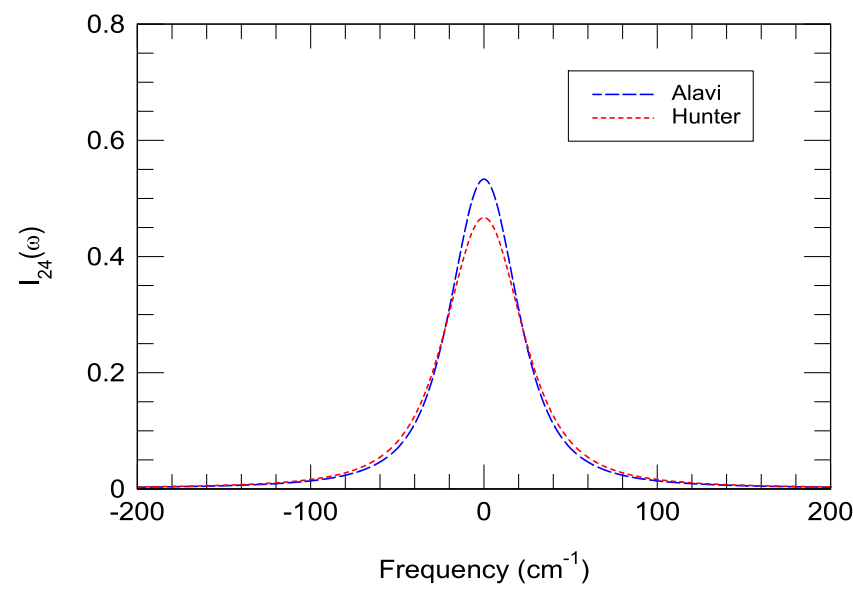

Figure 7. Fourier transform of $C_{24}(t)$, denoted here as $I_{24}(\omega)$, computed with potential models $V_{\text {hunter }}$ and $V_{\text {Alavi }}$ and $Q=0.485 e a_{0}^{2}$.

$j=0-3$ for a solution of molecular hydrogen in water with a molar ratio of $1 / 432$. Such a density ratio corresponds to a pressure of $14.7 \mathrm{MPa}$ [6]. It is close to the pressure of

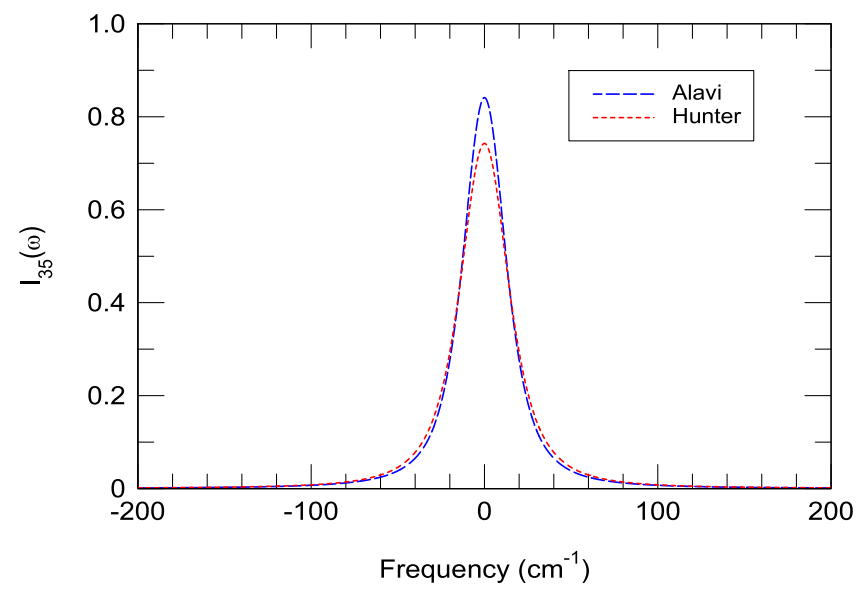

Figure 8. Fourier transform of $C_{35}(t)$, denoted here as $I_{35}(\omega)$, computed with potential models $V_{\text {hunter }}$ and $V_{\text {Alavi }}$ and $Q=0.485 e a_{0}^{2}$.

13.8 $\mathrm{MPa}$ at which the widths of the rotational lines were measured [4] and non-adiabatic calculations were performed [13]. At first we made our calculations with the same intermolecular potential as in [13]. Particularly, the anisotropic component of the $\mathrm{H}_{2}-\mathrm{H}_{2} \mathrm{O}$ potential has the same form as in equation (11) with $\langle 0|Q| 0\rangle=0.466 e a_{0}^{2}$ and $V_{\text {Hunter }}$ is the model for the isotropic component $\bar{v}_{00}$. The comparison of our results with those reported in [13] are presented in table 1 . In the first row of table 1 the experimental widths are reported for the first four $S_{0}(j)$ rotational lines [1, 3]. In the following rows we compare the widths calculated with our method and with the non-adiabatic method (see table 2 in [13]) using the same $\mathrm{H}_{2}-\mathrm{H}_{2} \mathrm{O}$ potential.

The comparison of the two numerical results shows an agreement between the two methods within $10 \%$, which we judge to be very satisfactory considering the relative simplicity of our approach. Moreover, our calculations describe experimental results qualitatively well. To this regard, it is important to remember here that we have used a multi-polar interaction to represent the anisotropic component of the $\mathrm{H}_{2}-\mathrm{H}_{2} \mathrm{O}$ interaction, which is a reasonable approximation only at large intermolecular distances.

In table 2, we report the results for the widths obtained with our method with three different $\mathrm{H}_{2}-\mathrm{H}_{2} \mathrm{O}$ potentials. The potential models are specified in the first column; $V_{\text {Hunter }}$ and $V_{\text {Alavi }}$ refer to the isotropic component. The anisotropic component of the $\mathrm{H}_{2}-\mathrm{H}_{2} \mathrm{O}$ potential energy is determined by the value of $\langle 0|Q| 0\rangle$ as shown by equation (11).

Table 2 shows the dependence of the Raman widths as a function of the isotropic and anisotropic components of the $\mathrm{H}_{2}-\mathrm{H}_{2} \mathrm{O}$ potential. While keeping the anisotropic component constant and switching from the model $V_{\text {Hunter }}$ [4] to the model $V_{\text {Alavi }}$ [7], the widths decrease. This is a result of the differences between the models used; model $V_{\text {Hunter }}$ has a smaller $\sigma$ and a larger $\epsilon$ than the model $V_{\text {Alavi }}$. For such a reason the potential model $V_{\text {Hunter }}$ allows the water molecules to stay closer to the hydrogen molecules than the potential model $V_{\text {Alavi }}$. Therefore, in the former case, the hydrogen molecules experience a more intense anisotropic interaction. 
Table 1. Comparison of measured HWHM, HWHM calculated with our method and with a non-adiabatic method using the same potential model.

\begin{tabular}{lcccc}
\hline Method & $S_{0}(0) \mathrm{cm}^{-1}$ & $S_{0}(1) \mathrm{cm}^{-1}$ & $S_{0}(2) \mathrm{cm}^{-1}$ & $S_{0}(3) \mathrm{cm}^{-1}$ \\
\hline experiment [1] & $47.6 \pm 0.6$ & $39.7 \pm 1.1$ & $28.0 \pm 1.4$ & $20.6 \pm 1.5$ \\
this work $\left(V_{\text {Hunter }},\langle 0|Q| 0\rangle=0.466\right)$ & 51.2 & 45.4 & 24.7 & 15.6 \\
non-adiabatic [13] & 50.4 & 41.5 & 22.5 & 15.3 \\
\hline
\end{tabular}

Table 2. HWHM calculated with our method with three different hydrogen-water potential models.

\begin{tabular}{lcccc}
\hline Method & $S_{0}(0) \mathrm{cm}^{-1}$ & $S_{0}(1) \mathrm{cm}^{-1}$ & $S_{0}(2) \mathrm{cm}^{-1}$ & $S_{0}(3) \mathrm{cm}^{-1}$ \\
\hline Experiment [1] & $47.6 \pm 0.6$ & $39.7 \pm 1.1$ & $28.0 \pm 1.4$ & $20.6 \pm 1.5$ \\
$V_{\text {Alavi }},\langle 0|Q| 0\rangle=0.485$ & 45.9 & 42.9 & 23.1 & 14.9 \\
$V_{\text {Hunter }},\langle 0|Q| 0\rangle=0.485$ & 54.4 & 48.3 & 26.3 & 16.7 \\
$V_{\text {Hunter }},\langle 0|Q| 0\rangle=0.466$ & 51.2 & 45.4 & 24.7 & 15.6 \\
\hline
\end{tabular}

Table 3. HWHM as a function of relative molar densities and of the model for the isotropic potential. $\langle 0|Q| 0\rangle=0.485 e a_{0}^{2}$.

\begin{tabular}{lccccc}
\hline$n_{H_{2} / n_{H_{2} O}}$ & $v_{00}$ model & $\begin{array}{c}S_{0}(0) \\
\mathrm{cm}^{-1}\end{array}$ & $S_{0}(1) \mathrm{cm}^{-1}$ & $\begin{array}{l}S_{0}(2) \\
\mathrm{cm}^{-1}\end{array}$ & $\begin{array}{l}S_{0}(3) \\
\mathrm{cm}^{-1}\end{array}$ \\
\hline $1 / 432$ & $V_{\text {Hunter }}$ & 54.4 & 48.3 & 26.3 & 16.7 \\
$5 / 432$ & $V_{\text {Hunter }}$ & 57.1 & 50.7 & 27.9 & 17.8 \\
$9 / 432$ & $V_{\text {Hunter }}$ & 60.3 & 53.4 & 29.5 & 19.1 \\
$1 / 432$ & $V_{\text {Alavi }}$ & 45.9 & 42.3 & 23.1 & 14.8 \\
$5 / 432$ & $V_{\text {Alavi }}$ & 48.6 & 44.9 & 24.7 & 15.9 \\
$9 / 432$ & $V_{\text {Alavi }}$ & 51.8 & 47.5 & 26.5 & 17.3 \\
\hline
\end{tabular}

Moreover, the comparison of the last two rows of table 2 shows that the widths decrease when we increase the value of the hydrogen quadrupole moment.

A comparison of the numerical and experimental results shows that the experimental widths for the $S_{0}(0)$ and $S_{0}(1)$ lines are best reproduced when using the model $\mathrm{V}_{\text {Alavi }}$ [7] for the isotropic component. $S_{0}(2)$ and $S_{0}(3)$ line widths are reproduced somewhat better by the $V_{\text {Hunter }}$ model. Taking into account that according to $[1,3]$, the $S_{0}(0)$ and $S_{0}(1)$ line widths were measured with higher precision than the $S_{0}(2)$ and $S_{0}(3)$ line widths, we are inclined to favour the $\mathrm{V}_{\text {Alavi }}$ with respect to the $\mathrm{V}_{\text {Hunter }}$ model. This finding is in agreement with conclusions about the vibrational $Q$ branch in [23].

In table 3, we report the widths as a function of the molar ratio $n_{\mathrm{H}_{2}} / n_{\mathrm{H}_{2} \mathrm{O}}$ both for potential models $V_{\text {Hunter }}$ and $V_{\text {Alavi }}$ and with $\mathrm{Q}=0.485 e a_{0}^{2}$.

Table 3 shows that, for both $V_{\text {Hunter }}$ and $V_{\text {Alavi }}$ models, the HWHM for every rotational line increases when the relative molar density, and thus the pressure of the solution, increases.

Finally, in figure 9, we report the computed total rotational Raman spectrum at the lowest pressure calculated with potential model $V_{\text {Alavi }}$. It can be compared with the experimental results reported in [3], particularly figures 6 .

In conclusion, in this paper we have demonstrated that the numerical results of the non-adiabatic calculations for the widths of the rotational Raman lines of hydrogen in water,

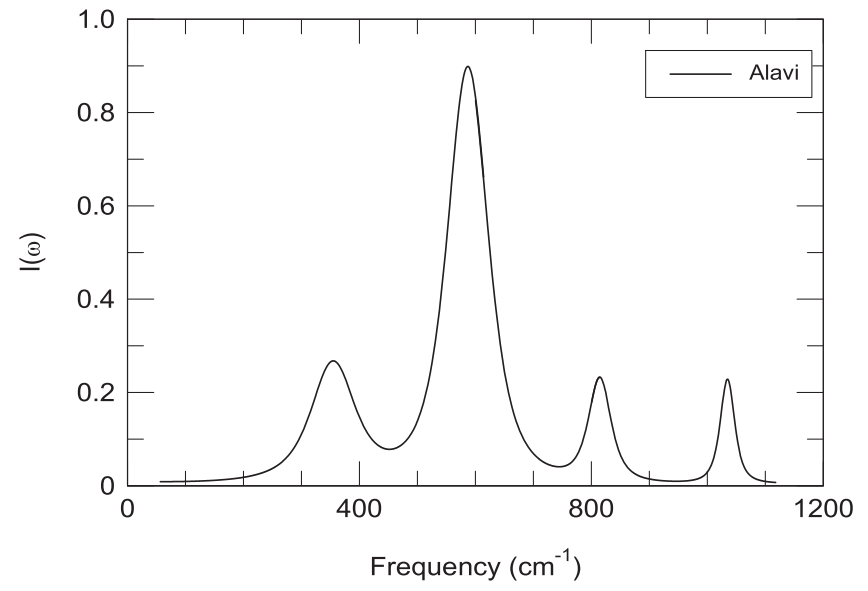

Figure 9. Rotational Raman spectrum with potential model 2 and $Q=0.485 e a_{0}^{2}$.

can be reproduced within $10 \%$ of accuracy by means of a method based on a perturbative expansion of the hydrogen polarizability correlation function. According to such method, the time dependence of the polarizability correlation function can be obtained by calculating time correlation functions of the anisotropic components of the $\mathrm{H}_{2}-\mathrm{H}_{2} \mathrm{O}$ intermolecular potential in a system in which the hydrogen molecules rotate freely. The effect of free rotations of the hydrogen molecules are easily calculated also in the quantum mechanical case. On the contrary, translations of the hydrogen molecules are coupled with roto-translational motion of the water molecules. This requires elaborate computations which can be performed, with a reasonable approximation, by means of classical molecular dynamics.

We have also shown how different models for the $\mathrm{H}_{2}-\mathrm{H}_{2} \mathrm{O}$ potential energy affect the shape of the rotational Raman lines. Particularly, we have considered the models of $[7,22]$ for the isotropic component and two different values for the hydrogen quadrupole moment for the anisotropic component.

Numerical results for the HWHM are also reported as a function of hydrogen concentration. 


\section{Acknowledgments}

Work supported in part by the National Science Fundation grant: ATM1625598

\section{ORCID iDs}

Jacek Borysow (iD https://orcid.org/0000-0002-9295-3567

\section{References}

[1] Taylor D J and Strauss H L 1989 J. Chem. Phys. 90768

[2] Taylor D J and Strauss H L 1992 J. Chem. Phys. 963367

[3] Chen Z, Lin Y-Y and Strauss H L 2000 J. Phys. Chem. B 1043274

[4] Hunter J E, Taylor D G and Strauss H L 1992 J. Chem. Phys., J. Chem. Phys. 9750

[5] Ziparo C, Giannasi A, Ulivi L and Zoppi M 2011 Int. J. Hydrog. Energy 367951

[6] Borysow J, Del Rosso L, Celli M, Moraldi M and Ulivi L 2014 J. Chem. Phys. 140164312

[7] Alavi S et al 2005 J. Chem. Phys. 123024507

[8] Alavi S, Klug D D and Ripmeester J A 2008 J. Chem. Phys. 128064506
[9] Valiron P, Wernli M, Faure A, Wiesenfeld L, Rist C, Kedzuch S and Noga J 2008 J. Chem. Phys. 129 134306

[10] Barreto P R B, Albernaz A F, Capobianco A, Palazzetti F, Lombardi A, Grossi G and Aquilanti V 2012 Comput. Theor. Chem. 99053

[11] Homayoon Z, Conte R, Qu C and Bowman J M 2015 J. Chem. Phys. 143084302

[12] Futera Z, Celli M, del Rosso L, Burnham C J, Ulivi L and English N J 2017 J. Phys. Chem. C 1213690

[13] Xiao L and Coker D F 1995 J. Chem. Phys. 1021107

[14] Mei H S, Xiao L and Coker D F 1996 J. Chem. Phys. 105 3938

[15] Kirk Veirs D and Rosenblatt G M 1987 J. Mol. Spectrosc. 121401

[16] Davies R W, Tipping R H and Clough S A 1982 Phys. Rev. A 263378

[17] Borysow A and Moraldi M 1989 Phys. Rev. A 401251

[18] Berendsen H J C, Grigera J R and Straatsma T P 1987 J. Phys. Chem. 916269

[19] DeCosmo V, Gush H P and Halpern M 1984 Can. J. Phys. 621713

[20] Hunt J L, Poll J D and Wolniewicz L 1984 Can. J. Phys. 621719

[21] Silvera I F and Goldman V V 1978 J. Chem. Phys. 694209

[22] Bickes R W Jr. et al 1975 J. Phys. B: At. Mol. Phys. 8 3034

[23] Borysow J, Moraldi M and Neumann M 2018 J. Phys. B: At. Mol. Opt. Phys. 51235101 